\title{
The Effect of Texting on Balance and Temporospatial Aspects of Gait
}

\author{
Emma C. Goddard ${ }^{1}$, Pierce T. Remler ${ }^{1}$, Robert H. Roos ${ }^{1}$, Robbie Turchyn ${ }^{1}$ \\ University of Western Ontario
}

\begin{abstract}
The purpose of this study was to determine effects of texting on standing balance and the temporospatial aspects of gait. It was hypothesized that texting would decrease stride velocity and increase deviation from midline while walking, as well as increase centre of pressure (CoP) excursions while standing. Fifteen participants (eleven males and four females, $21.12 \pm 1.25$ years of age) performed two standing balance tasks and two walking tasks. A repeated measures experimental design was used. The standing task consisted of standing as still as possible on a force plate for 20 seconds, which was then repeated while the participant texted a standard text message (48 characters). The second task consisted of walking along a six metre straight line while being filmed posteriorly along the line of progression, and perpendicular to direction of motion. This task was completed again while texting a similar predetermined message (48 characters). Balance performance was quantified by the percentage of total time within a $5 \mathrm{~mm}$ radius of each participant's centre of pressure (CoP). Gait quality was quantified using the average step length, stride velocity, and mediolateral standard deviation from midline averaged from both feet. The percentage of time spent within $5 \mathrm{~mm}$ of the average CoP was significantly $(p<0.05)$ less while texting compared to the non-texting control condition. Additionally, the mediolateral standard deviation from the midline while walking increased significantly $(p<0.05)$ in the texting condition. Average step length and average stride velocity decreased significantly $(p<0.05)$ while texting compared to the control condition. Standing balance and temporospatial aspects of walking are significantly degraded by texting. These results are valuable due to the growing prevalence of mobile technology. These results suggest that texting and walking could be detrimental to pedestrian safety and can help inform decisions regarding infrastructure to minimize potential dangers associated with distracted walkers.
\end{abstract}

KEYWORDS: biomechanics, gait, texting, temporospatial, kinesiology, balance

\section{Introduction}

Texting on a cell phone is a ubiquitous activity in today's society. In 2011, experts estimated that $77 \%$ of the world's population owned a mobile phone and that number has only grown in recent years (1). The dangers of texting and driving are common knowledge, but the effects of texting on walking are not as well known. Pedestrian safety is of large concern. In 2015, pedestrians constituted $15.2 \%$ of all Canadian Motor Vehicle Collisions fatalities and $14.3 \%$ of the serious injuries (2). Previous research has shown that texting can cause changes in postural balance (3), gait parameters (4$7)$, and walking behaviour $(1,3,4,8)$, which are attributed to the increased cognitive demand of performing two tasks at once.

Balance is defined as a continuous state in which the body reacts to internal and external perturbations to prevent a fall (9). Changes in balance and gait characteristics, such as stride velocity and medial-lateral deviation, could put pedestrians in danger (10). For example, an increase in medial-lateral deviation of stride could cause a pedestrian to step off a curb and into traffic or lack of visual information could cause a fall that results in a serious injury. In simulations, participants who texted while crossing a virtual street were more likely to display risky behaviours, and to be hit by vehicles, than non-texters (1). It has also been found that texters were more likely to misjudge the size and position of doorframes than non-texters (8). With the increasing prevalence of texting and walking in public places, it is important to understand the physical and behavioural changes associated with performing both tasks at once.

Previous research has investigated the impacts of texting on static and dynamic postural stability (11). Nurwulan et al. investigated static postural stability through normal and tandem stance with and without texting, and also evaluated dynamic balance through the use of the Star Excursion Balance Test (11). While the Star Excursion Balance Test is a valuable tool, it does not involve movements that are in accordance to activities of daily living (12). The present study included analysis of temporospatial gait parameters in order to evaluate more typical movement patterns.

The purpose of this study was to investigate the effects of texting on standing balance and gait characteristics while walking. It was hypothesized that texting would decrease step velocity and increase deviation from midline while walking, as well 
as increase centre of pressure (CoP) excursions during static balance.

\section{Methods}

\section{Participants}

This study was approved by the University of Western Ontario's Health Sciences Research Ethics Board. Fifteen participants (eleven males and four females) with an average age of $21.12 \pm 1.25$ years volunteered to be tested. All participants provided informed consent. Participants were recruited through poster advertisement. Participants were excluded if they had previous lower body, disabilities limiting mobility, or head injuries in the past 6 months. Disabilities that limit mobility and lower body injuries often produce compensatory gait patterns (13). Similarly, head injuries often produce cognitive changes that affect motor performance (14). The present study focused on normal, healthy gait only. Participants self-reported their frequency of texting while walking, and their perceived level of texting expertise. All participants were undergraduate or graduate students at the University of Western Ontario.

\section{Protocol}

Participants completed two tasks. In the first task, participants stood as still as possible on a piezoelectric force plate with their arms at their sides and their feet shoulder width apart. In the second task, the participant walked in a straight line while being filmed posteriorly along the line of progression and from the participants' left side, perpendicular to the direction of motion. The track used in the present study was $6.45 \mathrm{~m}$ long, allowing adequate length to produce a natural gait pattern (see Appendix). In a previous study, a track of $5.50 \mathrm{~m}$ allowed sufficient room for participants to accelerate to a normal gait speed, briefly maintain that speed then decelerate comfortably (15). The standing and walking tasks were both completed twice. The task was completed once as a control condition, where participants were not texting and then repeated while composing a standard text message (48 characters long) on their own mobile phone. All standing balance trials were 20 seconds long, which is a test duration long enough to reliably detect any changes in balance due to the test condition (16). The control condition was completed prior to the texting condition for both tasks.

\section{Equipment}

A piezoelectric force plate (9287B, Kistler Holding AG, Winterthur, Switzerland) was used to collect data regarding centre of pressure. The force plate was embedded in the floor of the lab. Voltage signals generated by the force plate were amplified (AMP 9865C, Kistler Holding AG, Winterthur, Switzarland), sampled at $100 \mathrm{~Hz}$ using a 16-bit analogue to digital conversion board (PCl 6221, National Instruments, Austin, Texas, USA) and recorded with a custom LabVIEW program (Version 10.0, National Instruments, Austin, Texas, USA).

Videos were recorded using two digital video cameras (Cyber-Shot SteadyShot DSC-W800, Sony, Tokyo, Japan) at 30 frames per second, with a shutter speed of $1 / 1500 \mathrm{~s}$. One camera was set posterior to the participant on a tripod $0.62 \mathrm{~m}$ high. The other camera was set on the left side of the participant, perpendicular to the participant's direction of motion $4.55 \mathrm{~m}$ away on a tripod $0.96 \mathrm{~m}$ in height.

\section{Analysis}

Voltages from the force plate were converted to forces and moments using customized LabVIEW software that implemented equations specific to the Kistler Equipment. These data were processed in Microsoft Excel (Version 14.6.2) to calculate CoP using the following equations:

$$
\begin{aligned}
& \text { 1. } x=-\frac{M_{y}+c F_{x}}{F z} \\
& \text { 2. } y=\frac{M_{x}-c F_{y}}{F_{z}}
\end{aligned}
$$

Where $\boldsymbol{c}$ is the location of origin of the force plate coordinate system, $\boldsymbol{x}$ is the location of centre of pressure in the $\boldsymbol{x}$ direction with respect to the geometric centre of the force plate, $\boldsymbol{y}$ is the location of the centre of pressure in the $\boldsymbol{y}$ direction with respect to the geometric centre of the force plate, $\boldsymbol{F}_{\boldsymbol{x}}$ is the medial-lateral force, $\boldsymbol{F}_{\boldsymbol{y}}$ is the anterior-posterior force, $\boldsymbol{F}_{\boldsymbol{z}}$ is the vertical force, $\boldsymbol{M}_{\boldsymbol{x}}$ moment about the $\mathrm{X}$ axis and $\boldsymbol{M}_{\boldsymbol{y}}$ is the moment about the $\mathrm{Y}$ axis.

The percentage of time spent in 4 rings (increasing in radius by $5 \mathrm{~mm}$ ) from the average CoP was calculated and compared between control and texting conditions using a paired t-test. Better balance was defined as maintaining CoP within a smaller area.

The video was analyzed using Kinovea Software (Version 0.8.15, www.kinovea.org). The video shot from the posterior produces a perspective change as the subject travelled further from the 
camera; therefore three objects of known length (located $1.00 \mathrm{~m}, 3.15 \mathrm{~m}$, and $5.30 \mathrm{~m}$ from the starting point) were input into Kinovea in order to calibrate measurements to account for this perspective change. Distance $(\mathrm{cm})$ of the foot from a midline that was placed along the path of movement was measured for each step in the video and exported to Microsoft Excel (Version 14.6.2). Standard deviation was calculated for both feet for each trial, and was averaged for both feet to allow for one measure of standard deviation per trial. A more consistent stride was defined as smaller standard deviation from midline.

The video shot from the viewpoint perpendicular to the direction of motion was also analyzed using Kinovea Software. One object of known length was used to calibrate measurements. Step length was defined as the distance between the toe of the leading foot and the toe of the trailing foot the instant before toe-off during double limb stance. Step length was averaged for each participant in each condition. Stride length was defined as distance between 2 successive placements of the same foot, consisting of 2 step lengths. Stride velocity (V) was calculated by dividing stride length by total stride time, which was based on the number of video frames. Dependent variables were analyzed using paired t-tests and all statistical analysis was performed using Microsoft Excel.

\section{Results}

There was significant difference in balance performance between control and texting conditions according to one-tailed, paired t-tests $(p<0.05)$. In the texting condition, the CoP spent significantly less time in the first ring, and significantly more time in rings two and three (Table 1, Figure 1).

When comparing medial-lateral standard deviation, there was significantly less deviation during the control condition $(p<0.01)$ (Table 2). Additionally, step length in the control condition was significantly longer, and stride velocity was significantly higher when compared to the texting condition (Table 2).

\section{Discussion}

The purpose of this study was to determine effects of texting on temporospatial aspects of gait and standing balance. It was hypothesized that texting would decrease stride velocity and increase deviation from midline while walking, as well as increase CoP excursions in quiet standing. CoP excursions would be represented by a larger percent of time spent outside a $5 \mathrm{~mm}$ radius from the average CoP.

The results of the present study support our hypotheses; gait and standing balance of the participants was significantly degraded by texting. These observed gait modifications are likely the result of the increased cognitive demands that are placed on working memory and executive control while performing dual tasks (17).

In agreement with the results from the present study, a previous study observed significant differences in static balance between the non-texting and texting conditions in which participants exhibited more sway in all parameters during texting conditions (10). This supports the notion that a secondary task impairs postural stability and indicates that the participants become more unstable while texting and therefore are more likely to fall (10).

Concurrent with a decrease in balance, all measured temporospatial parameters were adversely affected in the present study, including decreased velocity, increased deviation from midline, and decreased step length, further indicating that the distraction associated with cellular phone texting had a measurable effect on gait performance.

Previous studies supported the observations in this study, reporting a significant decrease in both step length and stride velocity $(4,5)$. It was suggested that participants engaged in cell phone use were unable to maintain walking speed or retain accurate spatial information during trials due to the inability to adequately divide attention between two tasks (4). It was theorized that this observation might be due to increased demands placed on the working memory system with the addition of a cell phone, resulting in gait disruptions (4).

In line with the results from the present study, previous studies found that lateral deviation while walking is significantly increased with the addition of texting $(4-6,18)$. It has been suggested that deviation from a straight path is the gait kinematic most likely to impact safety, potentially resulting in an increased likelihood of collisions, trips, and traffic accidents (6). If not regulated by central or peripheral vision, significant deviation from intended path can result in foot misplacement. This can lead to sprained ankles, falls, fractures, or worse if in high risk places like sidewalks or near stairs (6).

A field study found an increase in unsafe walking behaviours (i.e. sudden stops, disobeying traffic signals, and not looking both ways before crossing) among distracted individuals and more pronounced among those engaging in instant- 
messaging (18). In a controlled lab experiment using a virtual pedestrian environment, individuals that were texting were more likely to be struck by a virtual vehicle (1). The effects of texting on temporospatial aspects of gait and balance have implications for any user of a mobile phone when walking in a public environment.

\section{Limitations}

The current study evaluated quiet standing, a form of static balance, while texting. Walking, however, involves dynamic balance. Therefore, these findings may not be directly applicable to a dynamic balance scenario. The present study analyzed only two strides to determine stride velocity, which may not be representative of stride velocity over a longer walking length. A comment should be made about the difference in position of the participants arms between conditions. It has been shown that arm swing may improve the local dynamic stability of the trunk segments in mediallateral directions (19). Texting requires a participant to walk without arm swing with their arms held in front of their body, this could be a confounding variable. Also, the participants in this study were aware that they were being filmed, which may have affected their gait. Additionally, the use of Kinovea software to analyze step length and deviation from midline from video introduces human error when selecting reference points by hand. However, previous studies comparing manual digitizing in Kinovea to infra-red motion analysis found no significant difference in results from the camera/Kinovea method when compared to an Infrared measurement system, which allows us to maintain reasonable confidence in data collected for the present study when not considering human digitizing error (20). The study design had participants complete the non-texting control conditions first, allowing them to become familiarized with experimental setup. This design may have limited the results and should be randomized in the future.

\section{Future Directions}

Future research should include participants with a wider range of ages, as well as even distribution of males and females in order to ensure that the results are applicable across these populations. Gait lab technology could be used to analyze gait more accurately through the use of a three-dimensional marker position information that is used to determine the orientation of body segments, instantaneous joint center locations, and joint angles. Also, toe clearance is an important aspect of gait and therefore should be included in future research due to its relevance regarding gait perturbations resulting in tripping/falling. Additionally, since walking and texting utilizes dynamic balance, it may also be beneficial to analyze centre of pressure movement during gait or during dynamic balance tasks in addition to static balance. Future research should consider comparing text message accuracy to parameters of gait to determine whether more errors in text correlate with more consistent gait.

\section{Conclusion}

In the present study, the participants were able to maintain their CoP in a smaller area in control condition compared to the texting condition. Additionally, participants in the texting condition walked more slowly and with smaller steps, which deviated more in the mediolateral plane. This is likely due to the division of attention between the surrounding environment and the phone. Since texting and walking is very prevalent in today's society, these results suggest that this behaviour poses significant risk to pedestrians and should be considered an unsafe behaviour.

\section{Tables and Figures}

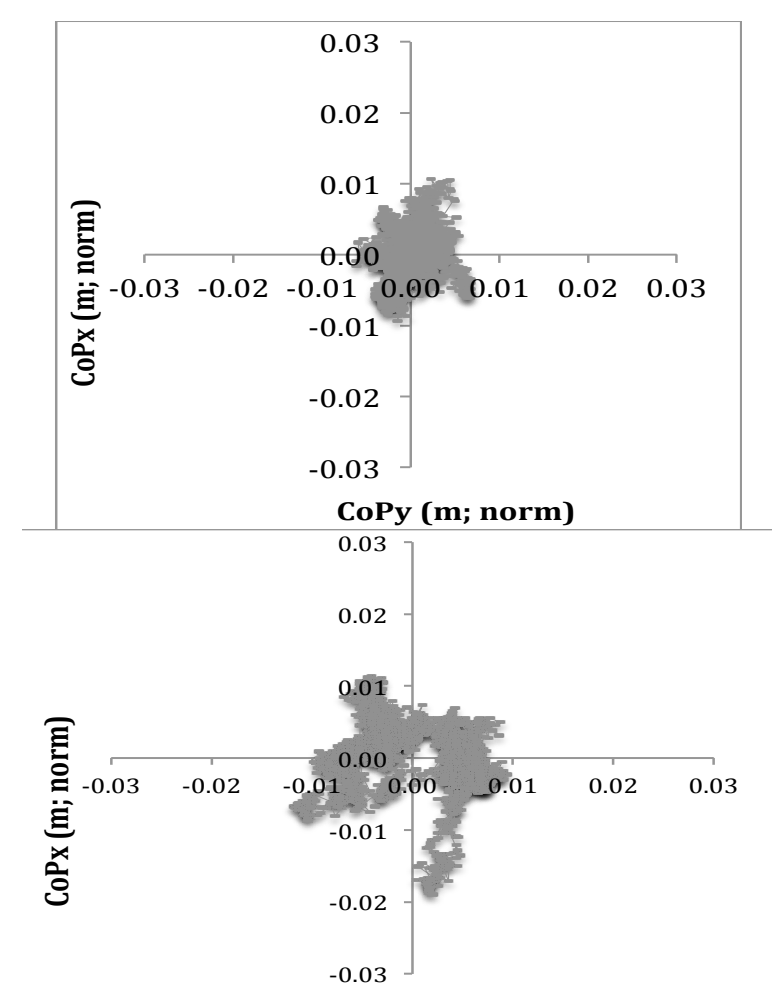

CoPy (m; norm) 
Figure 1: Excursions of CoP during static balance for both texting and control conditions. A) Excursions of CoP of one representative participant in control condition and B) Excursion of CoP of the same participant while texting.

Table 1. Percentage of total time spent with the participant's $\mathrm{CoP}$ in a $5 \mathrm{~mm}$ radius of their average $\mathrm{CoP} \pm$ standard deviation.

\begin{tabular}{|c|c|c|c|c|c|}
\hline \multirow{3}{*}{$\begin{array}{l}\text { Ring \# } \\
\text { Ring } 1(5 \mathrm{~mm})\end{array}$} & \multicolumn{5}{|c|}{ Average $\%$ of Time in Ring \pm Standard Deviation } \\
\hline & \multicolumn{2}{|c|}{ No Phone } & \multicolumn{2}{|c|}{ Phone } & \multirow{2}{*}{$\begin{array}{l}\text { P-Value } \\
0.017^{*}\end{array}$} \\
\hline & $70.02 \%$ & $\pm 16.26 \%$ & $53.89 \%$ & $\pm 23.99 \%$ & \\
\hline Ring $2(10 \mathrm{~mm})$ & $25.06 \%$ & $\pm 11.48 \%$ & $33.40 \%$ & $\pm 13.13 \%$ & $0.049 *$ \\
\hline Ring $3(15 \mathrm{~mm})$ & $4.24 \%$ & $\pm 5.59 \%$ & $9.98 \%$ & $\pm 10.47 \%$ & $0.046 *$ \\
\hline Ring $4(20 \mathrm{~mm})$ & $0.97 \%$ & $\pm 2.06 \%$ & $2.74 \%$ & $\pm 6.44 \%$ & 0.10 \\
\hline
\end{tabular}

${ }^{*}$ significantly different at $p<0.05$.

Table 2. Parameters of Gait for both texting and control conditions \pm standard deviation.

\begin{tabular}{lrllll}
\hline & \multicolumn{3}{c}{$\begin{array}{c}\text { Average } \pm \text { Standard Deviation } \\
\text { No Phone }\end{array}$} & Phone & P-Value \\
\hline Step Length $(\mathrm{m})$ & 1.02 & \pm 0.07 & 0.93 & \pm 0.08 & $6.39 \mathrm{E}-07^{*}$ \\
& 1.81 & \pm 0.17 & 1.53 & \pm 0.22 & $3.43 \mathrm{E}-05^{*}$ \\
$\begin{array}{l}\text { Stride Velocity }(\mathrm{m} / \mathrm{s}) \\
\begin{array}{l}\text { Mediolateral } \\
\text { Standard Deviation } \\
(\mathrm{cm})\end{array}\end{array}$ & & & & & \\
\hline * significantly different at $<0.01$. & 2.02 & \pm 0.87 & $3.70 \pm 1.09$ & $1.47 \mathrm{E}-05^{*}$ \\
\hline
\end{tabular}

\section{Appendix}

Experimental Lab Setup for Data Collection of Temporospatial Gait Parameters:

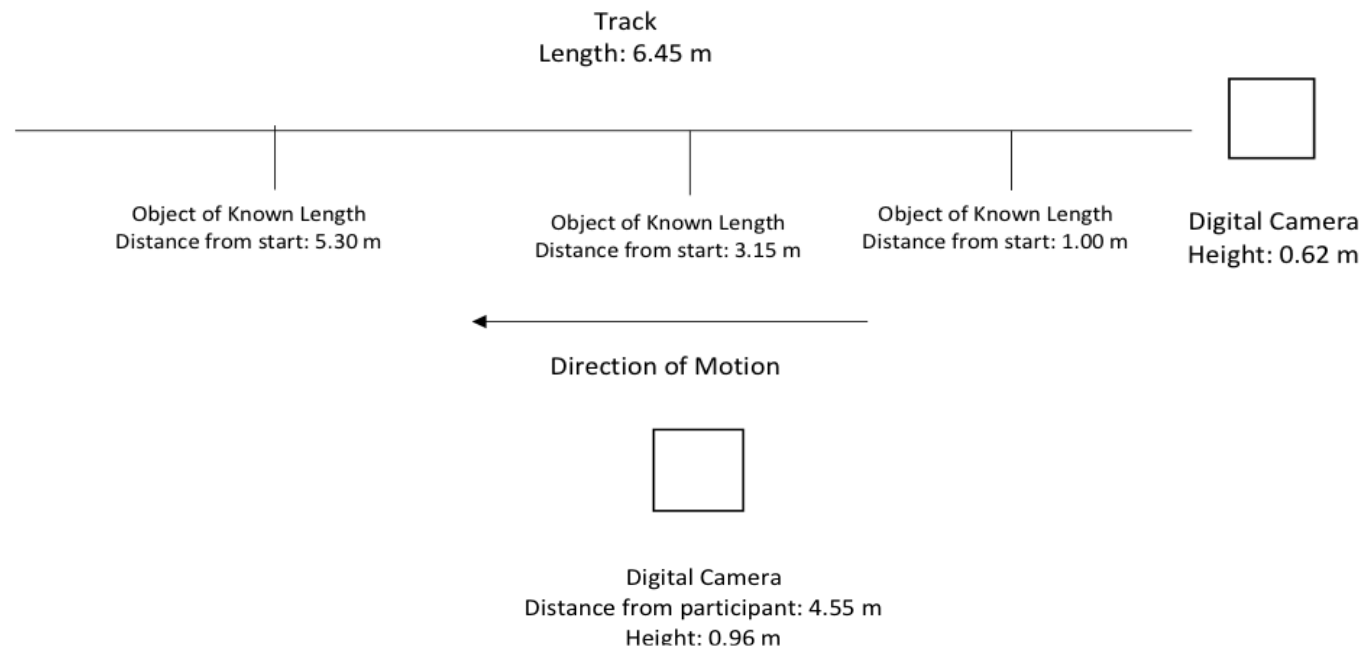




\section{References}

1. Schwebel DC, Stavrinos D, Byington KW, Davis T, O\&apos;Neal EE, De Jong D. Distraction and pedestrian safety: How talking on the phone, texting, and listening to music impact crossing the street. Accid Anal Prev [Internet]. 2012;45:266-71. Available from: http://dx.doi.org/10.1016/j.aap.2011.07.011

2. Transport Canada. Canadian Motor Vehicle Traffic Collision Statistics 2015 [Internet]. 2017. Available from:

http://www.tc.gc.ca/media/documents/roadsafe ty/TrafficCollisionStatisitcs_2011.pdf

3. Rebold MJ, Croall CA, Cumberledge EA, Sheehan TP, Dirlam MT. The impact of different cell phone functions and their effects on postural stability. Perform Enhanc Heal [Internet]. 2016; Available from: http://dx.doi.org/10.1016/j.peh.2016.11.004

4. Lamberg EM, Muratori LM. Cell phones change the way we walk. Gait Posture [Internet]. 2012;35(4):688-90. Available from: http://dx.doi.org/10.1016/j.gaitpost.2011.12.005

5. Licence S, Smith R, McGuigan MP, Earnest CP. Gait pattern alterations during walking, texting and walking and texting during cognitively distractive tasks while negotiating common pedestrian obstacles. PLoS One. 2015;10(7):1-11.

6. Parr ND, Hass CJ, Tillman MD. Cellular phone texting impairs gait in able-bodied young adults. J Appl Biomech. 2014;30(6):685-8.

7. Schabrun SM, Den Hoorn W, Moorcroft A, Greenland C, Hodges PW. Texting and walking: Strategies for postural control and implications for safety. PLoS One. 2014;9(1).

8. Lopresti-Goodman SM, Rivera A, Dressel C. Practicing Safe Text: The Impact of Texting on Walking Behavior. Appl Cogn Psychol. 2012;26(4):644-8.

9. Winter DA. Human blance and posture control during standing and walking. Gait Posture [Internet]. 1995;3(4):193-214. Available from: http://www.cs.cmu.edu/ hgeyer/Teaching/R16899B/Papers/Winter95Gait\%26Posture.pdf

10. Nurwulan NR, Jiang BC, Iridiastadi H. Posture and texting: Effect on balance in young adults. PLoS One. 2015;10(7):1-10.

11. Nurwulan NR, Jiang BC, Iridiastadi H. Posture and texting: Effect on balance in young adults. PLoS One. 2015;10(7):1-10.
12. Kinzey SJ, Armstrong CW. The Reliability of the Star-Excursion Test in Assessing Dynamic Balance. J Orthop Sport Phys Ther [Internet]. 1998;27(5):356-60. Available from: http://www.jospt.org/doi/10.2519/jospt.1998.27. 5.356

13. Shelburne KB, Torry MR, Pandy MG. Effect of muscle compensation on knee instability during ACL-deficient gait. Med Sci Sports Exerc. 2005;37(4):642-8.

14. Kuhtz-Buschbeck JP, Hoppe B, Gölge M, Dreesmann M, Damm-Stünitz U, Ritz A. Sensorimotor recovery in children after traumatic brain injury: analyses of gait, gross motor, and fine motor skills. Dev Med Child Neurol. 2003;45(12):821-8.

15. Oberg T, Karsznia A, Oberg K. Basic gait parameters: reference data for normal subjects, 10-79 years of age. J Rehabil Res Dev [Internet]. 1993;30(2):210-23. Available from:

http://www.rehab.research.va.gov/jour/93/30/2/ pdf/oberg.pdf

16. Gray VL, Ivanova TD, Garland SJ. Reliability of center of pressure measures within and between sessions in individuals post-stroke and healthy controls. Gait Posture [Internet]. 2014;40(1):198-203. Available from: http://dx.doi.org/10.1016/j.gaitpost.2014.03.191

17. Rubinstein JS, Meyer DE, Evans JE. Executive Control of Cognitive Processes in Task Switching. J Exp Psychol Hum Percept Perform. 2001;27(4):763-97.

18. Chen P-L, Saleh W, Pai C-W. Texting and walking: a controlled field study of crossing behaviours and inattentional blindness in Taiwan. Behav Inf Technol [Internet]. 2016;0(0):1-11. Available from: https://www.tandfonline.com/doi/full/10.1080/01 44929X.2016.1240234

19. Wu Y, Li Y, Liu AM, Xiao F, Wang YZ, Hu F, et al. Effect of active arm swing to local dynamic stability during walking. Hum Mov Sci. 2016;45:102-9.

20. Balsalobre-Fernandez C, Tejero-Gonzalez CM, Del Campo-Vecino J, Bavaresco N. The Concurrent Validity and Reliability of a LowCost, High-Speed Camera-Based Method for Measuring the Flight Time of Vertical Jumps. J Strength Cond Res. 2014;28(2):528-33. 